\title{
INFLUENCE OF AN ACCUMULATIVE ROLL BONDING (ARB) PROCESS ON THE PROPERTIES OF AA5083 AL-MG ALLOY SHEETS
}

\author{
Ana Alil ${ }^{1}$, Miljana Popović ${ }^{2 *}$, Tamara Radetic ${ }^{2}$, Endre Romhanji ${ }^{2}$ \\ ${ }^{I}$ Innovation Center of the Faculty of Technology and Metallurgy, University of \\ Belgrade, Belgrade, Serbia \\ ${ }^{2}$ Faculty of Technology and Metallurgy, University of Belgrade, \\ Belgrade, Serbia
}

Received 30.10.2014

Accepted 28.11.2014

\begin{abstract}
In this study, fully annealed AA5083 type alloy sheets with $1 \mathrm{~mm}$ in thickness were processed by accumulative roll bonding (ARB) at room temperature, up to 6 ARB cycles. It was found that microstructure was refined and mechanical properties were significantly improved with ARB processing. The maximum achieved values of hardness and tensile strength were two and three times greater than that of the initial material, respectively. This was attributed to the intensive strain hardening and grain size refinement which occurred during ARB deformation. However, the uniform elongation values were decreased by increasing the number of ARB cycles, and early fracture was registered. SEM fractography of fractured surfaces after tensile tests revealed a typical ductile fracture of ARB processed specimens, which was changed with ARB deformation. It was observed that ductile area on the fractured surfaces and the amount of necking, which occured before fracture, were decreased with increasing the number of ARB cycles.

Keywords: Accumulative roll bonding, Al-Mg alloy, mechanical properties, fracture surface
\end{abstract}

\section{Introduction}

Non heat treatable Al-alloys, such as Al-Mg alloys of 5xxx series, have a great potential for application in the transportation industry, due to the low density $(3 \times$ lower than in a steel), good weldability, corrosion resistance and ability to be recycled [1-3]. One of the important requests for their application in constructions of the transportation vehicles is providing a high strength and good formability. Grain size refinement is one

*Corresponding author: Miljana Popović, miljana@tmf.bg.ac.rs 
of the strengthening mechanisms, which also contributes to improvements in toughness, corrosion resistance and superplastic behavior at elevated temperatures [4].

With conventional methods of thermo-mechanical processing (TMP), which usually represent a combination of deformation by rolling and annealing, it is impossible to get microstructure with grain size below 15-20 $\mu \mathrm{m}$. Severe plastic deformation (SPD) processes, such as equal channel angular pressing (ECAP), high pressure torsion (HPT), accumulative roll bonding (ARB), etc. represent some of nonconventional processing methods, which can provide microstructure refinement till submicron level, and significant improvement of the strength [4,5]. Accumulative roll bonding (ARB) process is one of the most promising SPD methods to be implemented in the industry, as it uses the same equipment as conventional rolling. ARB process is developed by Saito [6,7] and up to date, many Al based alloys were ARB processed and used for investigation. However, most of them underwent ARB processing at elevated temperatures [8-10].

The aim of this paper was to investigate possibilities for room temperature ARB processing of Al-Mg AA5083 type alloy, and to examine its influence on the mechanical properties and fracture behavior of studied alloy.

\section{Experimental work}

Material. The material used in this study was industrially produced as a standard AA5083 alloy with the chemical composition given in Table 1. AA5083 alloy was supplied by Impol Seval Aluminium Rolling Mill, Sevojno, Serbia, in the form of hot rolled plates with an initial thickness of $10.6 \mathrm{~mm}$.

Table 1. Chemical composition of the studied and standard AA5083 type alloys (wt\%)

\begin{tabular}{cccccccccc}
\hline Alloy & $\mathrm{Mg}$ & $\mathrm{Mn}$ & $\mathrm{Cu}$ & $\mathrm{Fe}$ & $\mathrm{Si}$ & $\mathrm{Cr}$ & $\mathrm{Zn}$ & $\mathrm{Ti}$ & $\mathrm{Al}$ \\
\hline Studied & 4.16 & 0.47 & 0.0076 & 0.288 & 0.149 & 0.0599 & 0.0182 & 0.0086 & rest \\
\hline AA5083 & $4.0-4.9$ & $0.4-1.0$ & $<0.1$ & $<0.4$ & $<0.4$ & $0.05-0.25$ & $<0.25$ & $<0.15$ & rest \\
\hline
\end{tabular}

Further thermo mechanical processing of the alloy was performed in the laboratory according to the schematic illustration shown in Fig. 1.

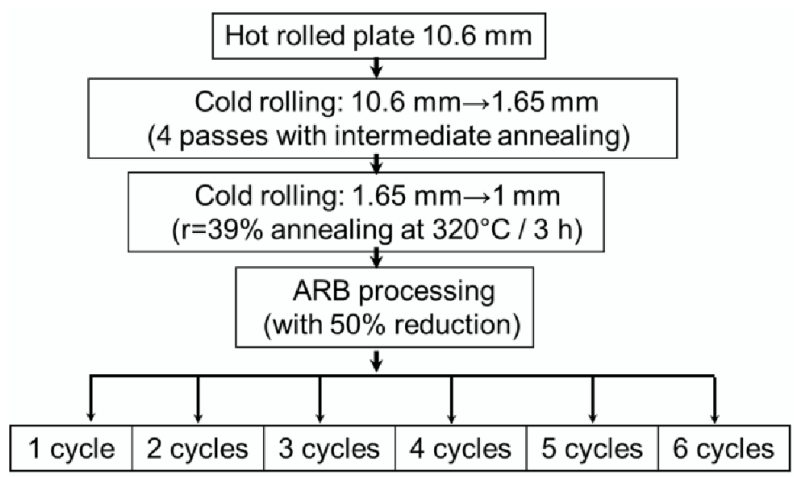

Fig. 1. Schematic illustration of thermo-mechanical processing of studied Al-Mg alloy. 
The as-received hot rolled specimens underwent laboratory cold rolling, from $10.6 \mathrm{~mm}$ to $1.65 \mathrm{~mm}$ in 4 passes (with $30-45 \%$ reductions), with recrystallization intermediate annealing. In order to produce $\mathrm{Al}-\mathrm{Mg}$ alloy sheets for accumulative roll bonding (ARB) process, final cold rolling was performed with a reduction of $39 \%$ $(1.65 \mathrm{~mm} \rightarrow 1 \mathrm{~mm})$, and the sheets were subsequently annealed at $320^{\circ} \mathrm{C} / 3 \mathrm{~h}$.

Accumulative roll bonding. The specimens for ARB processing, with a dimension of $1 \times 100 \times 160 \mathrm{~mm}$ (thickness $\times$ width $\times$ length) and a length parallel to rolling direction $(\mathrm{RD})$, were cut from the original annealed sheets. Two sheets with the same dimensions were stacked together and bonded by cold rolling with a reduction of approximately $50 \%$ in thickness (Fig. 2).

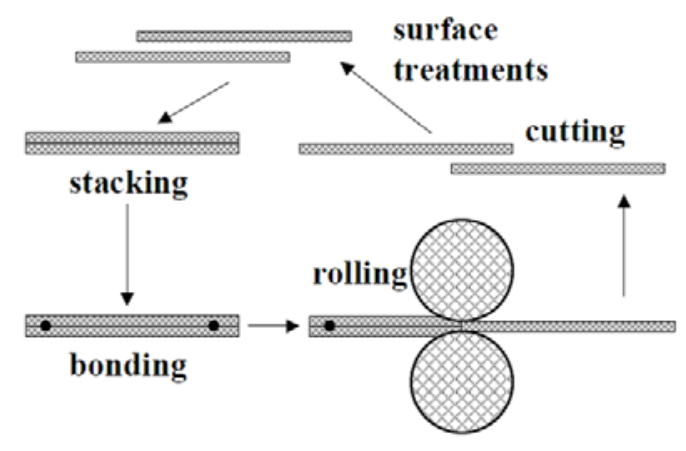

Fig. 2. Schematic illustration of accumulative roll bonding (ARB) process [5].

Before ARB deformation, one side of each specimen was prepared by degreasing, wire brushing, cleaning by methanol and air drying. The time between surface treatment and cold roll bonding was short $(<1 \mathrm{~min})$ in order to avoid formation of oxide layer on the surface. The ARB process was performed at room temperature, using a $206 \mathrm{~mm}$ diameter roll-mill at a peripheral rolling speed of $\sim 70 \mathrm{rpm}$. Prior to each ARB cycle, the rolls were cleaned by acetone and cold rolled bonding was performed under dry conditions. After each ARB cycle, the material was annealed in a furnace at $320^{\circ} \mathrm{C}$ for $5 \mathrm{~min}$, then cleaned and roll bonded. The procedure was proceeded for maximum $6 \mathrm{ARB}$ cycles and accumulative strain of about $\varepsilon=4.8$ was achieved.

Microstructure observations. Characterization of the microstructure was conducted by means of optical microscopy (OM) with a Reichert-Jung MeF3 optical microscope, in the longitudinal cross-section. Specimens were prepared by mechanical grinding, fine polishing, and electrochemical etching with Barker's reagent at a voltage of $20 \mathrm{~V}$. The grain structure was revealed under polarized light.

Resistivity measurements. The conductivity measurements were performed using Sigmatest 2.069 equipment, at operating frequency of $f=240 \mathrm{kHz}$, and conductivity was converted to resistivity values.

Mechanical properties. Mechanical properties were tested by hardness measurements and tensile testing. Brinell hardness of the specimens before and after each ARB cycle was measured under a load of $125 \mathrm{~kg}$ with the ball diameter $5 \mathrm{~mm}$. Tensile tests were conducted at room temperature using an INSTRON 1332 testing machine with an initial strain rate of $8.3 \times 10^{-4} / \mathrm{s}$. Tensile specimens, with the gauge 
width of $12.5 \mathrm{~mm}$ and a length of $50 \mathrm{~mm}$, were machined with the longitudinal direction parallel to the rolling direction.

Fractography. Fracture surfaces of the tensile specimens were observed by means of scanning electron microscopy (SEM) with a MIRA3 TESCAN microscope.

\section{Results}

\section{Microstructure}

The starting materials for ARB processing were annealed $\mathrm{Al}-\mathrm{Mg}$ sheets with a thickness of $1 \mathrm{~mm}$. Optical micrograph of the specimens before ARB shows fully recrystallized structure with average grain size of $23.13 \pm 0.29 \mu \mathrm{m}$ (Fig. 3a).
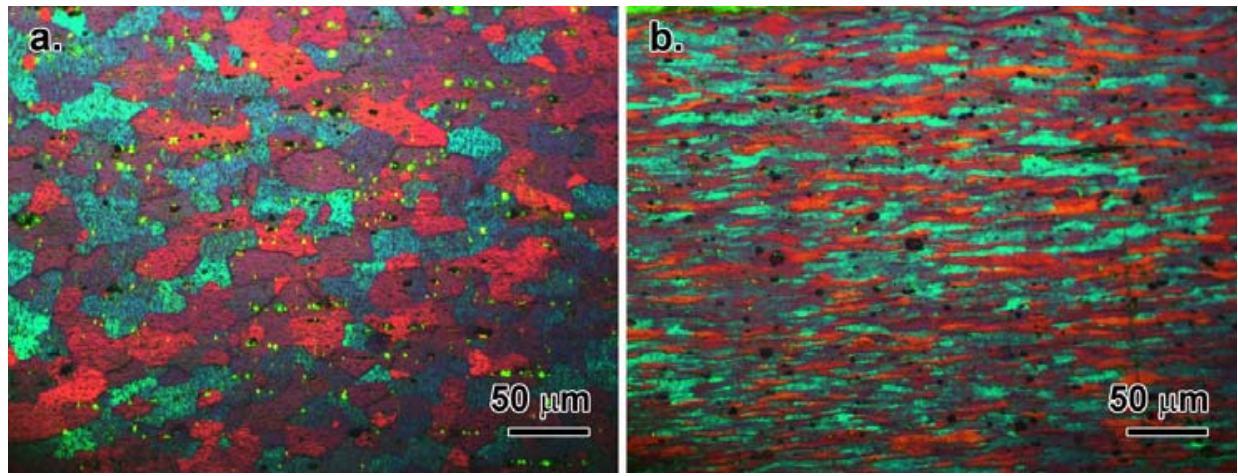

Fig. 3. Optical micrographs of tested Al-Mg alloy sheets (a) before ARB process, and (b) after 6 ARB cycles.

Microstructure of the specimens after 6 ARB cycles showed the grains elongated in the rolling direction, while the grain thickness became significantly reduced, as shown in Fig. 3b.

\section{Geometry of ARB processing}

During ARB processing, two processes occurs in parallel: plastic deformation and bonding of two sheets. In the case of tested Al-Mg alloy it was possible to achieve large plastic deformation, as shown in Fig. 4a.

Total rolling reduction in thickness $(r)$ was increased from $50 \%$ in the first ARB cycle up to $98 \%$ after $6 \mathrm{ARB}$ cycle, which resulted in an equivalent strain $\left(\varepsilon_{e q}\right)$ raise from 0.8 to 4.8 , i.e. about 0.8 per cycle. Total reduction $(r)$ and equivalent strain $\left(\varepsilon_{e q}\right)$ were calculated according to the following equations [11]:

$$
\begin{aligned}
& r=1-\frac{1}{2^{N}} \\
& \bar{\varepsilon}_{e q}=\frac{2}{\sqrt{3}} \cdot N \cdot \ln \frac{1}{1-r}=0.8 \cdot N
\end{aligned}
$$

where, $r$ is the reduction in thickness per cycle, and $N$ is the number of ARB cycles. 

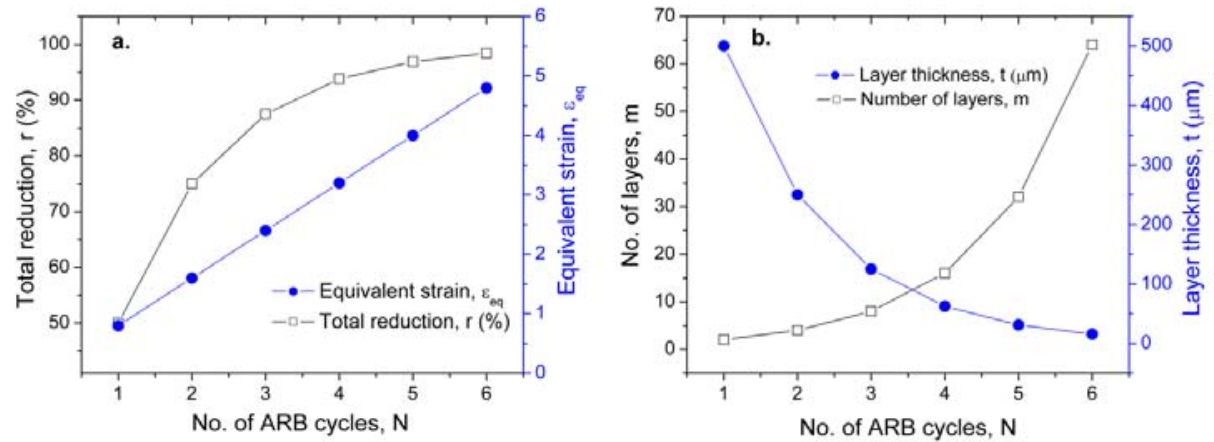

Fig. 4. Geometrical changes of tested Al-Mg alloy sheets during ARB processing, which were $1 \mathrm{~mm}$ thick and further roll bonded up to 6 ARB cycles by $50 \%$ reduction in thickness per cycle.

Fig. $4 \mathrm{~b}$ shows that number of layers within $1 \mathrm{~mm}$ thick ARB processed sheet increases from 2 layers after the first ARB cycle up to 64 layers after 6 ARB cycles. Number of layers, $m$, in the ARB processed sheet can be calculated as $m=2^{\mathrm{N}}$, and a layer thickness is $t=\mathrm{t}_{0} / 2^{\mathrm{N}}$, where $N$ is the number of ARB cycles, and $t$ is the thickness of each layer after $N$ ARB cycles. Fig. $4 \mathrm{~b}$ shows that the thickness of each layer decreases from $500 \mu \mathrm{m}$ to approximately $15 \mu \mathrm{m}$ with increasing the number of ARB cycles from 2 to 6 . The formation of new interfaces between the layers and geometrical changes in the tested Al-Mg alloy sheets which occured as a consequence of ARB processing is schematically illustrated in Fig. 5.

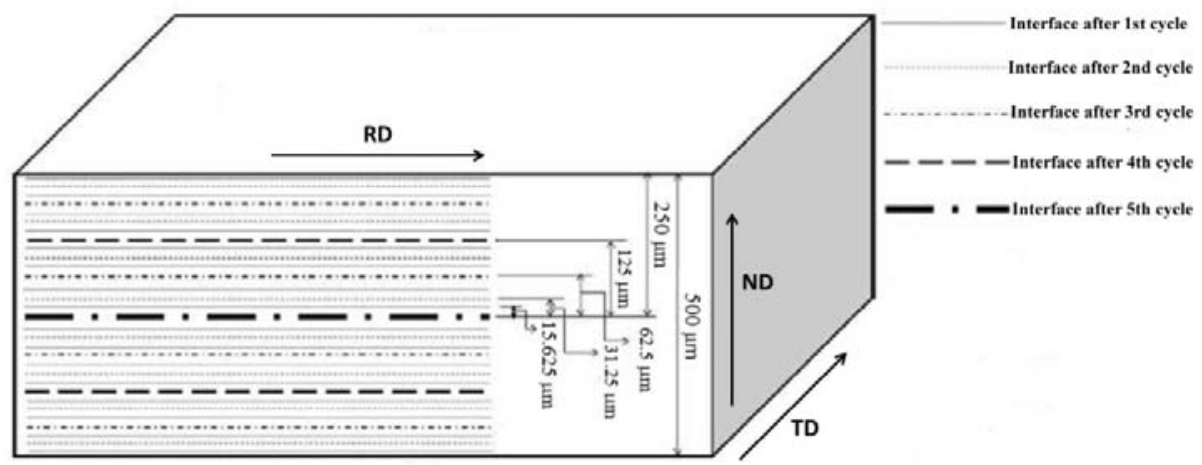

Fig. 5. Schematic illustration of interfaces between layers and its thickness after ARB deformation according to [12]. 


\section{Electrical resistivity measurements}

Electrical resistivity was used to follow microstructural changes which occured during ARB processing. Resistivity value is very sensitive to the presence of defects in the crystal lattice (vacancies, interstitials, dislocations, etc), as well as to the concentration of alloying elements in Al matrix/solid solution.

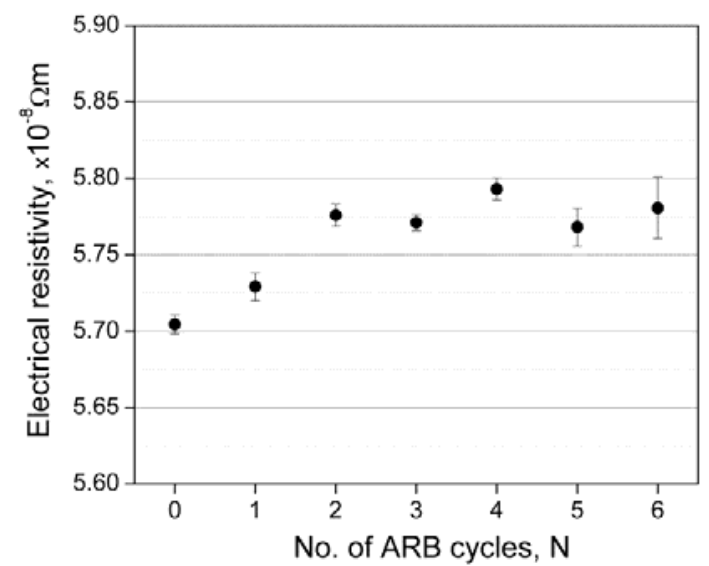

Fig. 6. Electrical resistivity variation with increasing the number of ARB cycles.

Figure 6 shows that electrical resistivity was slightly increased by an increase of the number of ARB cycles. Introducing total reduction in thickness of $98 \%$ after 6 ARB cycles, or plastic strain of $\varepsilon=4.8$, causes a resistivity change which was not more than $0.1 \times 10^{-8} \Omega \mathrm{m}$.

\section{Mechanical properties}

The average hardness (HB) values of the ARB processed samples are shown in Fig. 7a. The hardness of the starting fully annealed material, before ARB deformation, was about $\sim 60 \mathrm{HB}$. After ARB processing the hardness increases considerably after 1 ARB cycle and was increased almost twice $(\sim 115 \mathrm{HB})$ after 4 ARB cycles. Further $\mathrm{ARB}$ deformation caused a decrease of the hardness as it can be observed for the specimens after 5 and 6 ARB cycles (Fig. 7a).
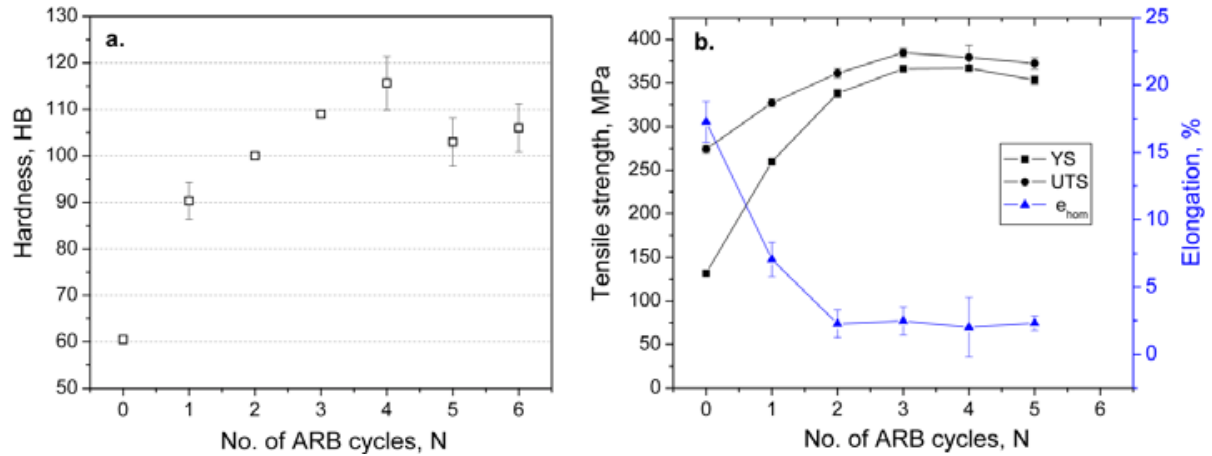

Fig. 7. Variation of (a) hardness (HB), and (b) tensile properties (YS, UTS and El) with increasing the number of ARB cycles 
Tensile properties of the tested Al-Mg sheets before and after ARB deformation are shown in Fig. 7b. The evolution of the tensile strength values with the number of ARB cycles is similar to that of the hardness values (Fig. 7a). Yield strength (YS) was increased rapidly after 1 and 2 ARB cycles, and the values after 3 and 4 ARB cycles were almost 3 times greater $(\sim 370 \mathrm{MPa})$ than that of the starting material $(\sim 130 \mathrm{MPa})$, before ARB deformation. The average values of ultimate tensile strength (UTS) were increased at a lower rate with increasing the number of ARB cycles, as shown in Fig. 7b. The UTS value increases from $\sim 275 \mathrm{MPa}$, in the initial state, to $\sim 385 \mathrm{MPa}$ after 3 and 4 ARB cycles. After 5 ARB cycles, a slight decrease of the YS and UTS values was observed. Figure $7 \mathrm{~b}$ shows that the average value of uniform elongation of ARB processed samples was substantially lower compared to the initial state of material. Uniform elongation was about $7.5 \%$ after $1 \mathrm{ARB}$ cycle, and decreased up to $2.5 \%$ after 2 ARB cycles. With further ARB deformation from 2 to 5 ARB cycles, it stayed almost unchanged.
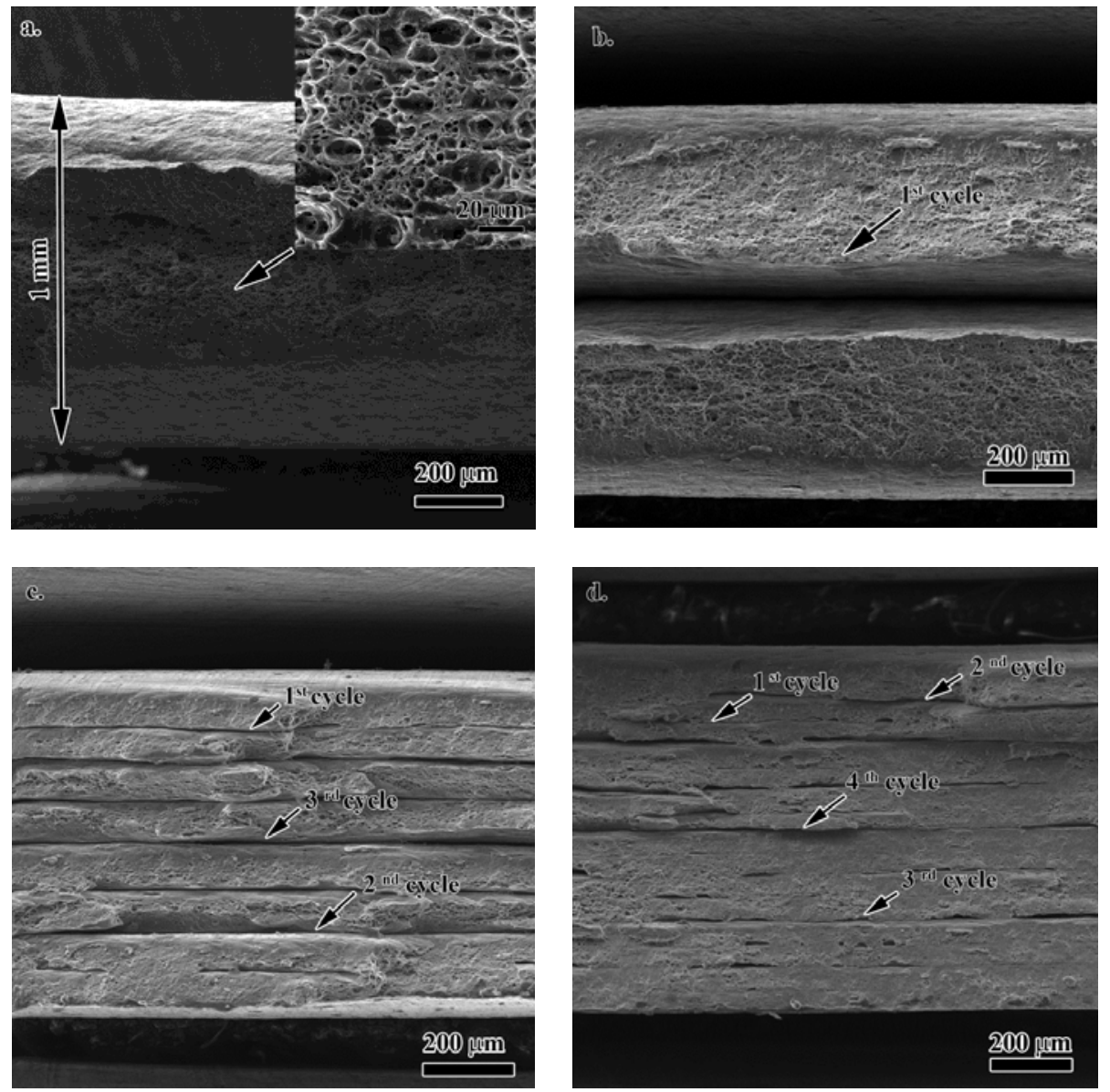

Fig. 8. Tensile fracture surface of tested Al-Mg alloy sheets (a) before ARB process, and after (b) first, (c) third, and (d) fourth ARB cycle. 


\section{Fractography}

Figure 8 shows tensile fracture surfaces of the specimens before and after ARB deformation. The fractograph in Fig. 8a shows that starting material, in the annealed state, exhibited typical ductile fracture that was preceded by large plastic deformation and a significant amount of necking before fracture. Tensile fracture surfaces of ARB processed specimens shows characteristics of ductile fracture, while more necking happened after 1 ARB cycle than after 3 or 4 ARB cycles, as shown in Fig. 8b-d. With increasing the number of ARB cycles, the fracture mode was changed and the amount of necking before fracture becomes smaller.

The fracture surface of the specimen after 4 ARB cycles was smooth and flat, with small area of ductile fracture, as shown in Fig. 8d. Tensile fracture surface was perpendicular to the tensile direction and necking area cannot be observed. The fracture surface after 4 ARB cycles shows a mixture of ductile and brittle fracture.

SEM fractographs of the ARB processed specimens in Fig. 8b-d also showed the individual stacked layers and interfaces between them. It is obvious that the number of interfaces was increased by increasing the number of ARB cycles. It can also be seen that better bonding was created between previous layers at higher ARB cycles, as shown in Fig. $8 \mathrm{~d}$ for the specimen after 4 ARB cycles. In that case the interface created after the first and second ARB cycle become almost invisible which indicated good and strong bonding between them.

\section{Discussion}

Results of this study showed that it was possible to conduct up to maximum 6 ARB cycles by processing of tested alloy at room temperature, while an equivalent plastic strain of $\varepsilon=4.8$ and total reduction in thickness of $98 \%$, were achieved (Fig. 4a). Such a large plastic deformation is not possible to introduce during conventional manufacturing of Al-plates and sheets by a combination of rolling and annealing. Previous studies of Saito [7] and Sheikh [13] showed that ARB processing of an AA5083 alloy at elevated temperatures $\left(t>200^{\circ} \mathrm{C}\right)$ was successful up to 7 ARB cycles. Toroghinejad et al [14] performed ARB processing of an AA5083 type alloy at ambient temperature and achieved 6 ARB cycles.

It is well known that accumulative roll bonding (ARB) is not only a deformation process performed by rolling, but also a bonding process of two stacked sheets. Number of layers within ARB processed sheet increases with increasing the number of ARB cycles, and the thickness decreases, as shown in Fig. 4b for tested alloy. Figure 5 illustrated a half of $1 \mathrm{~mm}$ thick Al-Mg alloy sheet after 6 ARB cycles, which consisted of 64 layers, with an average layer thickness of approximately $15 \mu \mathrm{m}$.

Besides geometrical changes, which occurred during ARB processing, it was shown that microstructure and mechanical properties were also affected by ARB deformation. Figure $3 \mathrm{a}$ showed that microstructure of the starting material before ARB deformation, consisted of recrystallized grains with an average grain size of $\sim 23 \mu \mathrm{m}$. The grain morphology was changed with ARB processing. The grains become elongated in the rolling direction, while a significant reduction in grain thickness occurred, as shown in Fig. $3 \mathrm{~b}$ for the specimen processed with 6 ARB cycles. It has been reported earlier $[6,8,14]$ that microstructure of ARB processed Al and Al-alloys can be refined till submicron level, with the grain size less than $500 \mathrm{~nm}$. 
Results of this study showed that ARB processing affected the grain morphology change, but also a resistivity level of tested alloy. Fig. 6 showed that electrical resistivity was slightly increased by an increase of ARB deformation, compared to the initial annealed state of material. The maximum increase in resistivity of about $0.1 \times 10^{-8}$ $\Omega \mathrm{m}$ was achieved, which can be attributed to the gradual increase in dislocation density and generation of dislocations during ARB deformation. Our previous study on the AlMg6.8 alloy [15] showed that an increase in deformation up to $70 \%$, performed by cold rolling, caused the same increase in resistivity of about $0.1 \times 10^{-8} \Omega \mathrm{m}$ due to generation of dislocations during cold rolling. However, it is worth noting that, with increasing the deformation up to $98 \%$, which was achieved by ARB processing of Al-Mg alloy studied in this paper, no further increase in resistivity was observed. This indicated that a saturation or steady-state in dislocation density may have occurred during ARB processing, possibly due to the activation of dynamic restoration mechanisms at high plastic strains. It is also interesting that multilayered structure and interfaces created between the layers did not have a significant effect on the resistivity values of ARB processed Al-Mg alloy sheets.

Geometrical and microstructural changes during ARB processing induced variations in the mechanical properties of the studied alloy, as shown in Fig. 7. It can be observed that hardness and tensile strength were increased rapidly after first ARB cycle, reached the maximum value at 3 or 4 ARB cycles, and then slightly decreased at high ARB cycles. The maximum average value of hardness became around two times greater than the value of starting material, with annealed coarse grained structure (Fig. 7a). The evolution of tensile strength showed that yield strength was almost three times greater after 3 or 4 ARB cycles, compared to the initial material (Fig. 7b). The ultimate tensile strength was increased at lower rate with increasing the number of ARB cycles, and improvement of approximately $30 \%$ can be observed in Fig. $7 \mathrm{~b}$. According to previous publications $[8,14,16,17]$, the increase in hardness and tensile strength was attributed to the strain hardening which occurred due to the accumulation of dislocation and its interactions during ARB deformation. On the other hand, we supposed that grain size refinement could also play an important role in the improvement of the mechanical properties through grain size strengthening mechanism. The strengthening effect reached the maximum level around 4 ARB cycles. The decrease in the hardness and strength at higher ARB cycles (Fig. 7b) can be attributed to the saturation or steadystate in dislocation density, which may have occurred due to dynamic restoration processes.

As shown in Fig. $7 \mathrm{~b}$, the uniform elongation of ARB processed samples was decreased rapidly from $\sim 17 \%$ for initial state to $\sim 7.5 \%$ after 1 ARB cycle, and to $\sim 2.5 \%$ after 2 ARB cycles. With further ARB deformation it stayed unchanged. Decreasing the ductility can be attributed to the intensive strain hardening which occurred through ARB processing, causing a decrease in dislocation mobility during tensile testing. Uniform elongation was also in good correlation with the variation of the difference between (UTS-YS), which decreasing with increasing the number of ARB cycles. This indicating that strain hardening capability became smaller and early fracture occurred in tensile testing. The obtained results on the mechanical properties evolution with the number of ARB cycles (high strength and low ductility) is the typical behavior for the materials with ultra fine grained structure, which were produced by ARB processing $[6,8,16,17]$. 
Tensile fracture surfaces of ARB processed specimens shows characteristics of ductile fracture, while the amount of necking before fracture become smaller with increasing the number of ARB cycles (Fig. 8b-d). On the other hand, it can be seen that number of the interfaces between layers increases, and according to previous studies $[14,16]$ they may represent a potential site for crack nucleation and propagation. Delamination or debonding between the layers can also contribute in decreasing the uniform elongation in tensile testing, as reported earlier [14,16]. Fig. $8 \mathrm{~d}$ shows that interfaces become less visible at higher ARB cycles, indicating better bonding. However, the strain hardening capacity was exhausted at high ARB cycles and the specimen showed low ductility, becoming less ductile in tensile testing.

\section{Summary}

In this study, the effect of accumulative roll bonding (ARB) process on the properties of AA5083 Al-Mg alloy sheets was investigated, and experimental results showed that:

- ARB processing at room temperature was possible to perform up to 6 ARB cycles, and large equivalent plastic strain of $\varepsilon=4.8$ was achieved;

- Grain morphology was changed and substantially refined, while mechanical properties were improved through ARB deformation. The maximum value of hardness became greater twice, and yield strength was increased almost three times, in comparison with that of the material before ARB. The increase in mechanical properties can be attributed to the strain hardening and grain size strengthening, while a slight decrease in the hardness and strength, was supposed to occur due to dynamic restoration processes;

- Low ductility in tensile testing of the specimens processed with more than two ARB cycles was due to intensive strain hardening. The ductile fracture surface area, which is characteristics of ARB processed specimens, and the amount of necking before fracture was decreased with increasing the number of ARB cycles.

\section{Acknowledgement}

The authors are grateful to the Ministry of Education, Science and Technological Development of The Republic of Serbia, and Impol Seval Aluminium Rolling Mill, Sevojno, Serbia, for supporting this research under contract number TR 34018.

\section{References}

[1] G.B. Burger, A.K. Gupta, P.W. Jeffrey, D.J. Lloyd, Mater. Character. 35 (1995) 23-39.

[2] W.S. Miller, L. Zhuang, J. Bottema, A.J. Wittebrood, P. De Smet, A. Haszler, A.Vieregge, Mater. Sci. Eng. A 280 (2000) 37-49.

[3] D.J. Lloyd, Mater. Forum, 28 (2004) 107-117.

[4] R.Z. Valiev, R.K. Islamgaliev, I.V. Alexandrov, Progr. Mater. Sci., 45 (2000) 103189.

[5] B. Verlinden, J. Metallurgy (MJoM), 11(3) (2005) 165-182.

[6] Y. Saito, N. Tsuji, H. Utsunomiya, T. Sakai, R.G. Hong, Scripta Mater., 39 (1998) 1221-1227.

[7] Y. Saito, H. Utsunomiya, N. Tsuji, T. Sakai, Acta Mater., 47 (1999) 579-583. 
[8] H. Pirgazi, A. Akbarzadeh, R. Petrov, L. Kestens, Mater. Sci. Eng. A497 (2008) 132-138.

[9] X. Huang, N. Tsuji, N. Hansen, Y. Minamino, Mater. Sci. Eng. A340 (2003) 265271.

[10] K.-T. Park, H.-J. Kwon, W.-J.Kim, Y.-S. Kim, Mater. Sci. Eng. A316 (2001) 145152.

[11] N. Tsuji, Y. Saito, S.H. Lee, Y. Minamino, Adv. Eng. Mater., 5 (2003) 338-344.

[12] Shibayan Roy, Nataraj B.R., Satyam Suwas, S. Kumar, K. Chattopadhyay, Mater. Design, 36 (2012) 529-539.

[13] H. Sheikh, Scripta Mater., 64 (2011) 556-559.

[14] M. R. Toroghinejad, F. Ashrafizadeh, R. Jamaati, Mater. Sci. Eng. A561 (2013) 145-151.

[15] M. Popović, E. Romhanji, Mater. Sci. Eng. A492 (2008) 460-467.

[16] L. Su, C. Lu, H. Li, G. Deng, K. Tieu, Mater. Sci. Eng. A614 (2014) 148-155.

[17] M. Eizadjou, H.D. Manesh, K. Janghorban, J. Alloy Comp., 474 (2009) 406-415. 